\title{
Inelastic Momentum Transfer Measurements on Plasmonic Structures in the (S)TEM
}

\author{
J Schultz $^{1 *}$, Krehl ${ }^{1}$, G Guzzinati ${ }^{2}$, P Potapov $^{1}$, FS Nogueira ${ }^{3}$ and A Lubk ${ }^{1}$ \\ 1. Institute for Solid State Research, IFW Dresden, Dresden, Germany. \\ 2. EMAT, University of Antwerp, Antwerp, Belgium. \\ 3. Institute for Theoretical Solid State Physics, IFW Dresden, Dresden, Germany. \\ * Corresponding author: j.schultz@ifw-dresden.de
}

Surface plasmons are self-sustaining resonances occurring at interfaces of dielectric media where the permittivity changes sign. They often exhibit strongly enhanced, localized electrical fields, so they couple strongly to electromagnetic, in particular optical, excitations. Accordingly, surface plasmons are able to be used for sub-wavelength control of electromagnetic fields. Based on this, novel electronic devices can be realized, for instance on-chip light spectrometers and linear accelerators, plasmonic rectennas for the harvesting of light or LEDs and photovoltaics with a higher efficiency.

For a thorough understanding of the properties of plasmonic resonances in different materials and structures the spatial distribution of transient electromagnetic fields is of particular interest. To map the evanescent electrical field with high spatial and spectral resolution, electron energy-loss spectroscopy (EELS) in the scanning transmission electron microscope (STEM) can be utilized. Here, on the one hand, the Cartesian z-component of the induced electric field parallel to the beam direction causes an energy loss of the beam electrons, on the other hand, the lateral components of the induced electric and magnetic field lead to a deflection of the electron beam.

Probing the z-component of the electric field by recording EELS spectra at different STEM-probe positions is a well-established technique called spectrum imaging (SI) [1]. Due to the lack of sensitivity to lateral electric field components and the induced magnetic field, some effects of plasmonic excitations are not detectable with SI or other techniques like scanning near-field optical microscopy (SNOM) or photo electron emission microscopy (PEEM). To overcome this constrain, we developed a novel technique in the STEM, called Inelastic Momentum Transfer (IMT), that allows the reconstruction of the lateral field components from an energy-filtered beam deflection measurement [2]. A schematics of the IMT setup is shown in Fig. 1.

Our proof of concept experiment enables us to reconstruct all three Cartesian components of the induced electric field strength in plasmonic nanoparticles (see Fig. 2) [2]. The access to lateral electric and magnetic field components via IMT can be employed in a number of applications. Here, we discuss the possibility to investigate the outstanding plasmonic properties of topological insulators, where topological field theory leads to axion electrodynamics. Within this theory the Lagrangian density is modified by a magnetoelectric axion-term $L_{a}=\alpha \theta /\left(4 \pi^{2}\right)$ with $\alpha$ the fine-structure constant and $\theta=\pi$ for time-reversal symmetries [3]. Analytic studies suggest, that there is no considerable change of the energy-loss due to the additional magnetoelectric axion-term. However, the modification of the induced magnetic field has an appreciable effect on the beam deflection which is potentially detectable with IMT measurements [4]. 


\section{References:}

[1] J Nelayah et al., Nature Physics 3(5) (2007), p. 348.

[2] J Krehl et al., Nature Communications 9(1) (2018), p. 4207.

[3] FS Nogueira and J van den Brink, arXiv e-prints, 1808.08825 (2018) (accessed Dec 20, 2018).

[4] We acknowledge funding from the European Research Council (ERC) under the Horizon 2020 research and innovation program of the European Union (grant agreement No 715620).

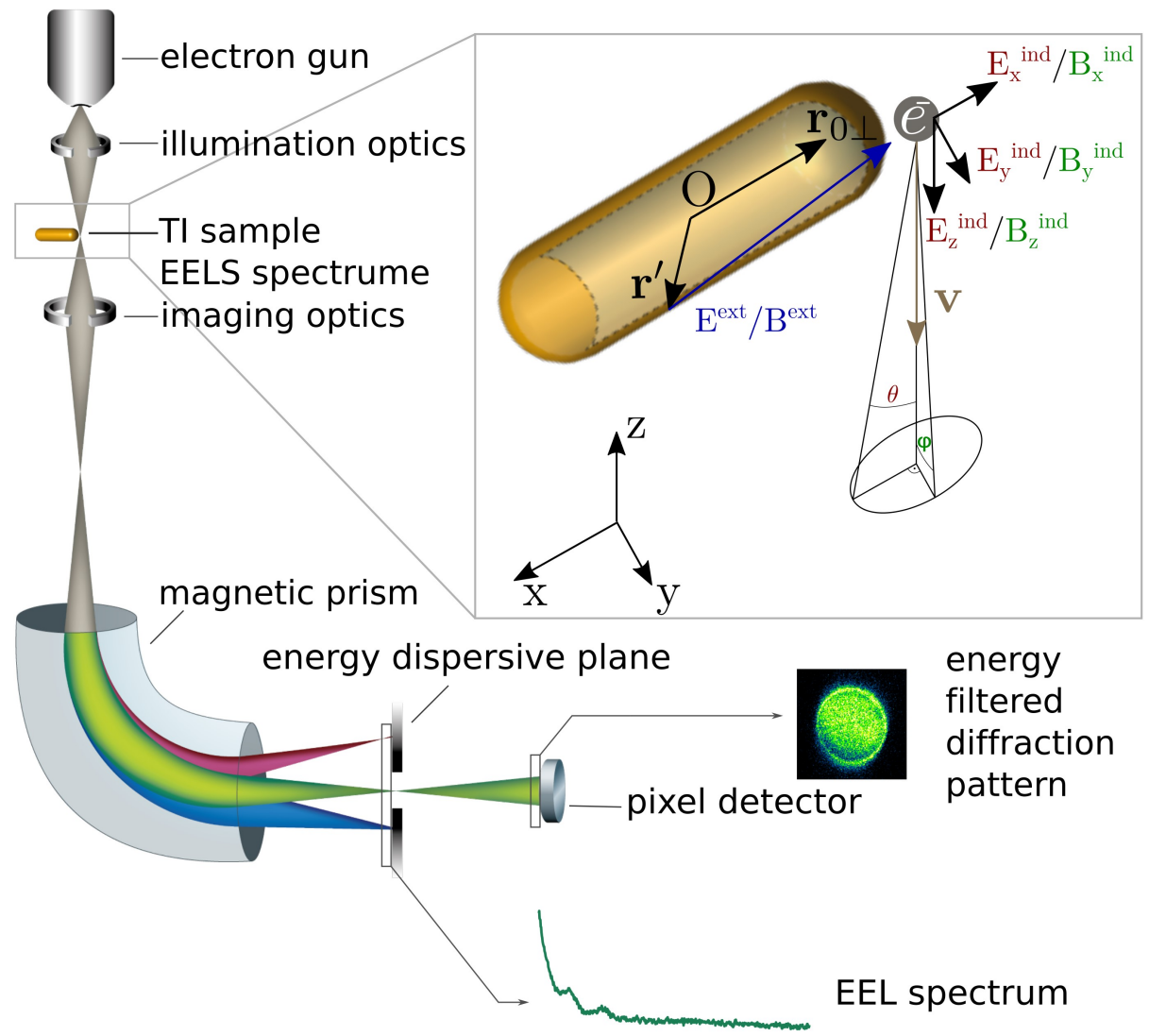

Figure 1. Experimental Setup of SI and IMT measurements. In the case of SI a EEL spectrum is recorded in the energy dispersive plane by a pixel detector at each STEM-probe position. For IMT measurements an energy selective slit is placed in the energy dispersive plane and an energy filtered diffraction pattern is recorded in the far field at each STEM-probe position. By evaluation of the intensity and the center of mass of the diffraction disks the beam deflection can be reconstructed.

a)

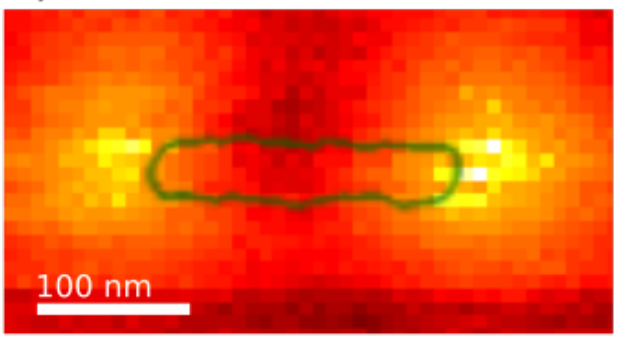

$\Gamma$ in $10^{-3} \mathrm{fs}$

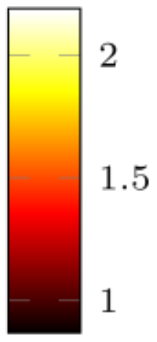

b)

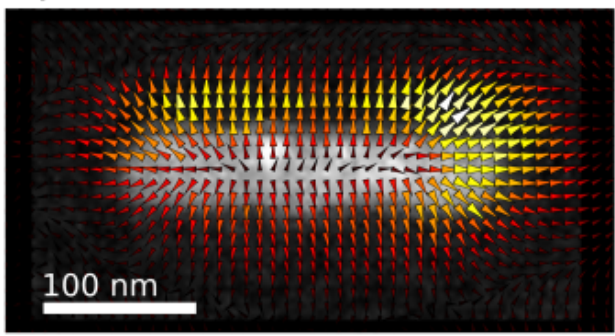

$\widetilde{\boldsymbol{E}}_{\perp}^{\text {ind }}$ in $\frac{\mathrm{MV} \mathrm{fs}}{\mathrm{m}}$

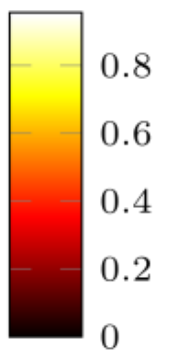

Figure 2. Reconstructed electric field components of a $200 \mathrm{~nm}$ aluminum nanorod. a) shows the energy loss probability corresponding to the z-component of the induced spectral field strength. b) depicts the lateral components of the induced spectral field strength. 